\title{
Effets de la forme de la rainure d'alimentation d'un palier sur l'écoulement hydrodynamique, le débit et la distribution de la pression
}

\author{
Loubna Jeddi $^{1}$, Mohamed El Khlifi $^{1, a}$ et Dominique Bonneau ${ }^{2}$ \\ 1 Université Hassan II - Mohammedia, faculté des sciences et techniques, UFR de mécanique des fluides et de structures, \\ BP 146, 20650 Mohammedia, Maroc \\ 2 Université de Poitiers, UMR CNRS 6610, Laboratoire de Mécanique des Solides, 4 avenue de Varsovie, \\ 16021 Angoulême Cedex, France
}

Reçu le 26 septembre 2003, accepté le 2 juillet 2004

\begin{abstract}
Résumé - Une procédure numérique est développée pour étudier les effets de la forme du raccordement rainure/coussinet (carrée, et arrondie avec différents rayons de courbure) d'un palier hydrodynamique sur le développement de l'écoulement, le débit et la distribution de la pression. Les équations bidimensionnelles de Navier-Stokes, écrites pour un écoulement laminaire d'un lubrifiant incompressible, sont résolues numériquement par la méthode des éléments finis en utilisant la méthode de chargement par incrémentation. La simulation numérique utilise la vitesse de l'arbre et la pression d'alimentation comme paramètres dynamiques, alors que le rayon de courbure de raccordement rainure/coussinet est utilisé comme paramètre géométrique. Le travail présenté dans cet article souligne que la pression d'alimentation, l'interaction de la couche de cisaillement avec l'écoulement dans la cavité et l'interaction du coussinet avec le domaine de la rainure à travers un raccordement carré ou arrondi influencent l'écoulement, aussi bien que la magnitude et le profil des pressions et des vitesses dans la rainure. Il est en particulier montré qu'un choix judicieux de profil de raccordement - à pression d'alimentation égale - permet de doubler l'apport de fluide frais dans le film lubrifiant.
\end{abstract}

Mots clés : Rainure hydrodynamique / lubrification / forme de la rainure / vitesse / pression / film lubrifiant

\begin{abstract}
Effects of feed journal bearing groove shape on the hydrodynamic flow, flow rate and pressure distribution. A numerical procedure is developed for study the effects of the shape of the groove/land joints (square, and rounded with different radii of curvature) of the hydrodynamic journal bearing on the development of the flow, flow rate and pressure distribution. The bidimensional NavierStokes equations, written for the laminar flow of an incompressible lubricant, are solved numerically using the incremental load method and the finite element formulation. The numerical simulation uses the shaft velocity and the feeding pressure as dynamic parameters, while the radii of curvature of the groove/land joints is used as a geometric parameter. The work presented in this paper points out that the feeding pressure, the interaction of the shear layer with the cavity flow and the interaction of the land with the groove domain through square or rounded shape influence the flow patterns, as well as the magnitude and the profile of the pressures and the velocities in the groove. It is particularly shown that a right choice of groove/land joints profile - in an equal feeding pressure case - allows a double fresh fluid outcome in the lubricant film.
\end{abstract}

Key words: Hydrodynamic groove / lubrication / groove shape / velocity / pressure / lubricant film

a Auteur correspondant : elkhlifi@eudoramail.com 


\section{Nomenclature}

\begin{tabular}{|lll|}
\hline$h$ & épaisseur du film dans les deux faces, gauche et droite, de la rainure, & $\mathrm{m}$ \\
$h_{\mathrm{r}}$ & profondeur de la rainure carrée & $\mathrm{m}$ \\
$h_{\mathrm{t}}$ & profondeur de l'orifice d'alimentation & $\mathrm{m}$ \\
$\ell_{\mathrm{r}}$ & largeur de la rainure & $\mathrm{m}$ \\
$\ell_{\mathrm{t}}$ & largeur de l'orifice d'alimentation & $\mathrm{m}$ \\
$p$ & pression & $\mathrm{Pa}$ \\
$P_{\mathrm{alim}}$ & pression d'alimentation & $\mathrm{Pa}$ \\
$q=\int_{0}^{h} u \mathrm{~d} y$ & débit unitaire & $\mathrm{m}^{2} \cdot \mathrm{s}^{-1}$ \\
$R_{\mathrm{e}}=\frac{\rho U_{\mathrm{arb}} h_{\mathrm{r}}}{\mu}$ & & \\
$u, v$ & nombre de Reynolds & \\
$U_{\mathrm{arb}}$ & composantes du vecteur vitesse & $\mathrm{m} \cdot \mathrm{s}^{-1}$ \\
$x, y$ & vitesse périphérique de l'arbre & $\mathrm{m} \cdot \mathrm{s}^{-1}$ \\
$\mu$ & coordonnées cartésiennes & $\mathrm{m}$ \\
$\rho$ & viscosité dynamique du lubrifiant & $\mathrm{Pa}^{-}$ \\
\end{tabular}

\section{Introduction}

Face aux exigences industrielles actuelles, le débit en lubrifiant des paliers doit être de mieux en mieux contrôlé. À cette fin, la modélisation de l'écoulement dans les poches hydrostatiques ainsi que dans les rainures hydrodynamiques des paliers devient nécessaire. Les simulations numériques aident généralement à la compréhension de la physique du mélange entre le fluide frais d'alimentation et le fluide recyclé, et à la prédiction de pertes de pression dues à l'inertie et ses effets sur la dynamique des paliers.

Si de nombreuses études ont été réalisées sur les performances et la définition géométrique des paliers en utilisant les équations de base de la mécanique des films minces visqueux $[1,2]$, très peu d'entre elles traitent le mécanisme particulier de l'écoulement dans les poches et les rainures, et encore moins leurs dimensionnement. Ettles [3] a donné une analyse détaillée de l'écoulement dans une rainure sans prendre en compte les effets hydrostatiques, pendant que Heckelman et Ettles [4] ont étudié les effets de la pression à l'entrée de la cavité du palier. Tichy et Bourgin [5] ont également mis en évidence les effets de la pression d'entrée à l'entrée du palier. Braun et al. $[6,7]$ ont confirmé de leur côté l'existence des pertes de pression sur les bords d'une poche hydrostatique. En outre, Dzodzo et al. [8] ont étudié le développement du champ hydrodynamique dans une poche hydrostatique peu profonde, avec différentes formes du raccordement poche hydrostatique/coussinet.

Pour ce travail, un code de calcul, basé sur la résolution des équations bidimensionnelles de NavierStokes discrétisées par la méthode des éléments finis, a été développé. Le modèle numérique retenu permet d'analyser les effets de la forme du raccordement rainure hydrodynamique/coussinet (carrée et arrondie avec différents rayons de courbure) sur l'écoulement et sur la distribution de la pression au niveau de l'arbre aussi bien dans le cas d'un effet de Couette dominant que dans le cas d'une pression d'alimentation dominante. L'influence de ce choix de profil sur l'apport en fluide frais dans le film sera particulièrement analysée.

\section{Description du problème}

Le schéma d'une rainure d'alimentation axiale d'un palier hydrodynamique est présenté sur la figure 1. Les caractéristiques géométriques sont données dans le tableau 1. Le paramètre physique principal qui différencie les diverses géométries de la rainure, présentées dans cette étude, est le rayon de la courbure $R$ du Raccordement Rainure/Coussinet (RRC). L'écoulement est laminaire, isotherme et stationnaire. Le lubrifiant est newtonien et incompressible. Les effets de la gravité sont négligés.

Les équations bidimensionnelles de continuité et de Navier-Stokes gouvernant l'écoulement s'écrivent :

$$
\begin{gathered}
\frac{\partial u}{\partial x}+\frac{\partial v}{\partial y}=0 \\
-\rho\left(u \frac{\partial u}{\partial x}+v \frac{\partial u}{\partial y}\right)+\mu\left(\frac{\partial^{2} u}{\partial x^{2}}+\frac{\partial^{2} u}{\partial y^{2}}\right)-\frac{\partial p}{\partial x}=0 \\
-\rho\left(u \frac{\partial v}{\partial x}+v \frac{\partial v}{\partial y}\right)+\mu\left(\frac{\partial^{2} v}{\partial x^{2}}+\frac{\partial^{2} v}{\partial y^{2}}\right)-\frac{\partial p}{\partial y}=0
\end{gathered}
$$

Afin de permettre une obtention précise du champ hydrodynamique, des conditions aux limites réalistes sont imposées aux limites du lubrifiant et du solide (coussinet, arbre).

Au niveau de l'arbre, la vitesse du lubrifiant est égale à $U_{\text {arb }}$.

Sur le coussinet, la condition d'adhérence est appliquée.

Sur la frontière d'entrée du trou d'alimentation, la composante $u$ du vecteur vitesse est nulle et la pression est égale à la pression d'alimentation du lubrifiant frais. La composante $v$ du vecteur vitesse est à calculer.

À chacune des frontières gauche et droite de la rainure, la composante $v$ du vecteur vitesse est nulle et la 
Tableau 1. Caractéristiques géométriques de la rainure.

\begin{tabular}{lccc}
\hline Paramètre & Symbole & Unités & Valeur \\
\hline Épaisseur du film dans les deux faces, & $h$ & $\mathrm{~m}$ & 0,0002 \\
gauche et droite, de la rainure & & & \\
Profondeur de la rainure carrée & $h_{\mathrm{r}}$ & $\mathrm{m}$ & 0,002 \\
Profondeur de l'orifice d'alimentation & $h_{\mathrm{t}}$ & $\mathrm{m}$ & 0,042 \\
Largeur de la rainure & $\ell_{\mathrm{r}}$ & $\mathrm{m}$ & 0,02 \\
Largeur de l'orifice d'alimentation & $\ell_{\mathrm{t}}$ & $\mathrm{m}$ & 0,016 \\
Viscosité dynamique du lubrifiant & $\mu$ & Pa.s & 0,04 \\
Masse volumique & $\rho$ & $\mathrm{kg} . \mathrm{m}^{-3}$ & 800 \\
\hline
\end{tabular}

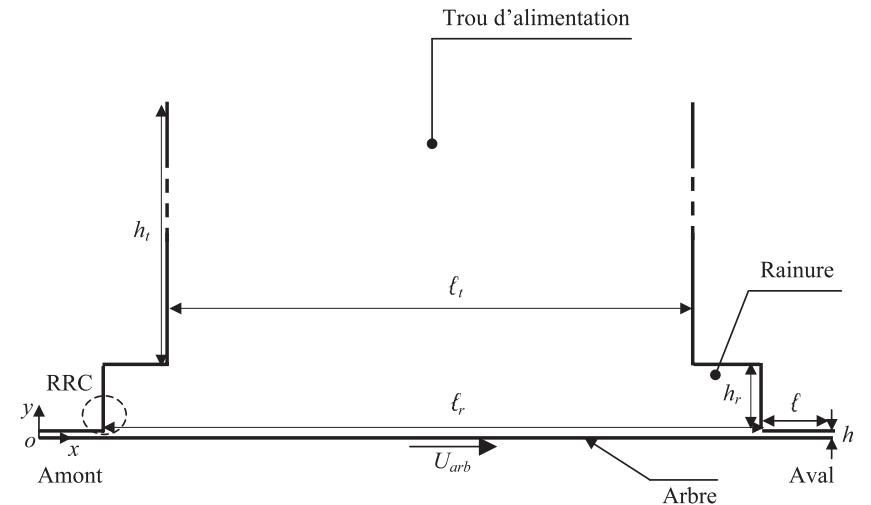

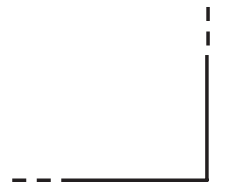

RRC carré

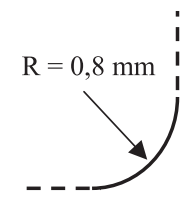

RRC arrondi

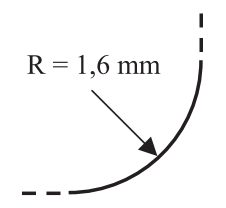

Fig. 1. Schéma d'une rainure d'un palier hydrodynamique.

pression est assignée à une pression de référence $(p=0)$. La composante $u$ du vecteur vitesse est à calculer.

L'analyse hydrodynamique est basée sur la résolution simultanée des équations (1)-(3) en tenant compte des conditions aux limites associées. La méthode des éléments finis [9] est appliquée sur tout le domaine d'étude occupé par le lubrifiant. Le choix des fonctions de pondération est basé sur la méthode de Bubnov-Galerkin [9]. Le maillage de ce domaine, raffiné au niveau de RRC, est composé d'éléments isoparamétriques à 8 nœuds. Les composantes $u$ et $v$ du vecteur vitesse sont interpolées à partir des huit valeurs nodales :

$$
\begin{aligned}
& u=\sum_{k=1}^{8} N_{k}^{u} u_{k} \\
& v=\sum_{k=1}^{8} N_{k}^{u} v_{k}
\end{aligned}
$$

où $N_{k}^{u}$ sont des fonctions quadratiques.
Par contre, et pour éviter les sur-contraintes, la pression $p$ est interpolée seulement à partir des quatre valeurs nodales de coin :

$$
p=\sum_{k=1}^{4} N_{k}^{p} p_{k}
$$

où $N_{k}^{p}$ sont des fonctions linéaires.

Le système d'équations discrétisées est non linéaire par rapport aux inconnues du problème $u, v$ et $p$. Il est résolu par la méthode de chargement par incrémentation associée à la méthode itérative de Newton-Raphson [10].

\section{Résultats et discussions}

Le paramètre géométrique retenu est le rayon de courbure de RRC, qui a une influence directe sur la physique de l'écoulement aussi bien dans le cas d'un effet de Couette dominant $\left(U_{\mathrm{arb}}=3,4 \mathrm{~m} \cdot \mathrm{s}^{-1}\left(R_{\mathrm{e}}=136\right)\right.$ et $\left.P_{\text {alim }}=0,04 \mathrm{MPa}\right)$ que dans le cas d'une pression d'alimentation dominante $\left(U_{\mathrm{arb}}=1 \mathrm{~m} \cdot \mathrm{s}^{-1}\left(R_{\mathrm{e}}=40\right)\right.$ et $\left.P_{\text {alim }}=0,2 \mathrm{MPa}\right)$.

\subsection{Influence de la densité du maillage}

L'effet de la densité du maillage sur le débit total, dans le cas par exemple d'une pression d'alimentation dominante $\left(U_{\text {arb }}=1 \mathrm{~m} \cdot \mathrm{s}^{-1}\right.$ et $\left.P_{\text {alim }}=0,2 \mathrm{MPa}\right)$ et pour un RRC carré, est présenté dans le tableau 2. Le débit total obtenu tend à être indépendant du maillage à partir de 464 éléments du maillage. Une observation de lignes de courant obtenues pour ces différents maillages ne montre pas de différences significatives. De ce fait, le choix d'un nombre d'éléments de l'ordre de 500 est suffisant pour prédire le comportement hydrodynamique du lubrifiant dans la rainure d'alimentation.

\section{2 Écoulements dans la rainure avec effet de Couette dominant $\left(U_{\text {arb }}=3,4 \mathrm{~m} . \mathrm{s}^{-1}\right.$ et $P_{\text {alim }}=0,04 \mathrm{MPa}$ )}

La figure 2 représente les lignes de courant dans la partie droite et dans la partie gauche de la rainure pour 

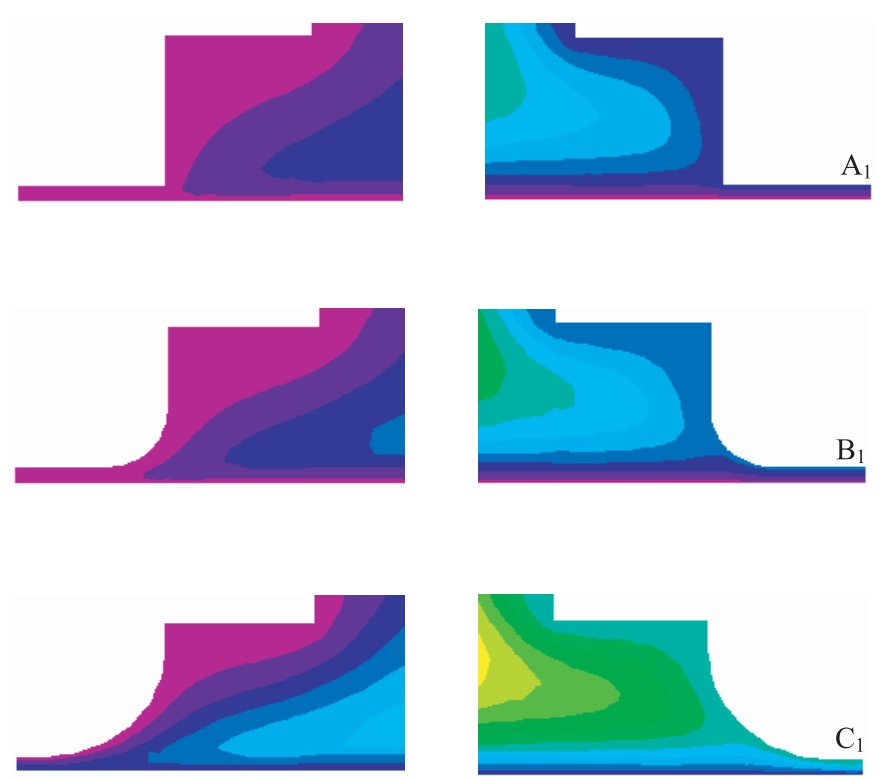

Sens de déplacement de la surface de l'arbre

Fig. 2. Lignes de courant dans la rainure pour différentes formes $\mathrm{du}$ raccordement rainure/coussinet $\left(U_{\mathrm{arb}}=3,4 \mathrm{~m} \cdot \mathrm{s}^{-1}\right.$ et $\left.P_{\text {alim }}=0,04 \mathrm{MPa}\right) . \mathrm{A}_{1}: \mathrm{RRC}$ carré, $\mathrm{B}_{1}: R=0,8 \mathrm{~mm}$ et $\mathrm{C}_{1}: R=1,6 \mathrm{~mm}$.

Tableau 2. Effet de la densité du maillage sur le débit total.

\begin{tabular}{cc}
\hline Nombre d'éléments & Débit total $\left(10^{-3} \mathrm{~m}^{2} \cdot \mathrm{s}^{-1}\right)$ \\
\hline 354 & 2,3155 \\
464 & 2,6544 \\
542 & 2,6545 \\
754 & 2,6545 \\
\hline
\end{tabular}

un RRC carré (Fig. 2A $\mathrm{A}_{1}$ ), arrondi avec $R=0,8 \mathrm{~mm}$ (Fig. $2 \mathrm{~B}_{1}$ ) et $R=1,6 \mathrm{~mm}$ (Fig. $2 \mathrm{C}_{1}$ ) respectivement. Les lignes de courant dans la partie centrale de la rainure et dans le trou d'alimentation sont illustrées par les figures $3 \mathrm{~A}_{1}-3 \mathrm{C}_{1}$. Généralement, on peut observer dans les régions de la partie droite de la rainure (Figs. $2 \mathrm{~A}_{1}-2 \mathrm{C}_{1}$ ) une zone de recirculation qui représente une excroissance du tourbillon qui peut être vu dans son intégralité dans les figures $3 \mathrm{~A}_{1}-3 \mathrm{C}_{1}$. Au fur et à mesure que le rayon de courbure de RRC augmente, la zone de recirculation située dans la partie droite de la rainure diminue légèrement en hauteur. Cette diminution est due à la quantité de fluide supplémentaire qui sort en aval. La partie gauche de la rainure, quant à elle, présente une zone de recirculation qui semble se déplacer vers le bas avec l'augmentation du rayon de courbure de RRC.

Sur la frontière gauche de la rainure, le lubrifiant est refoulé à contre-courant et l'influence du rayon de courbure $R$ est illustrée par un changement du profil de vitesse (Fig. 4). Au fur et à mesure que $R$ augmente, la quantité du fluide entraînée par l'arbre diminue et l'écoulement qui, à l'origine était entrant, devient de plus en plus sortant. La longueur du domaine d'entrée égale à celle du
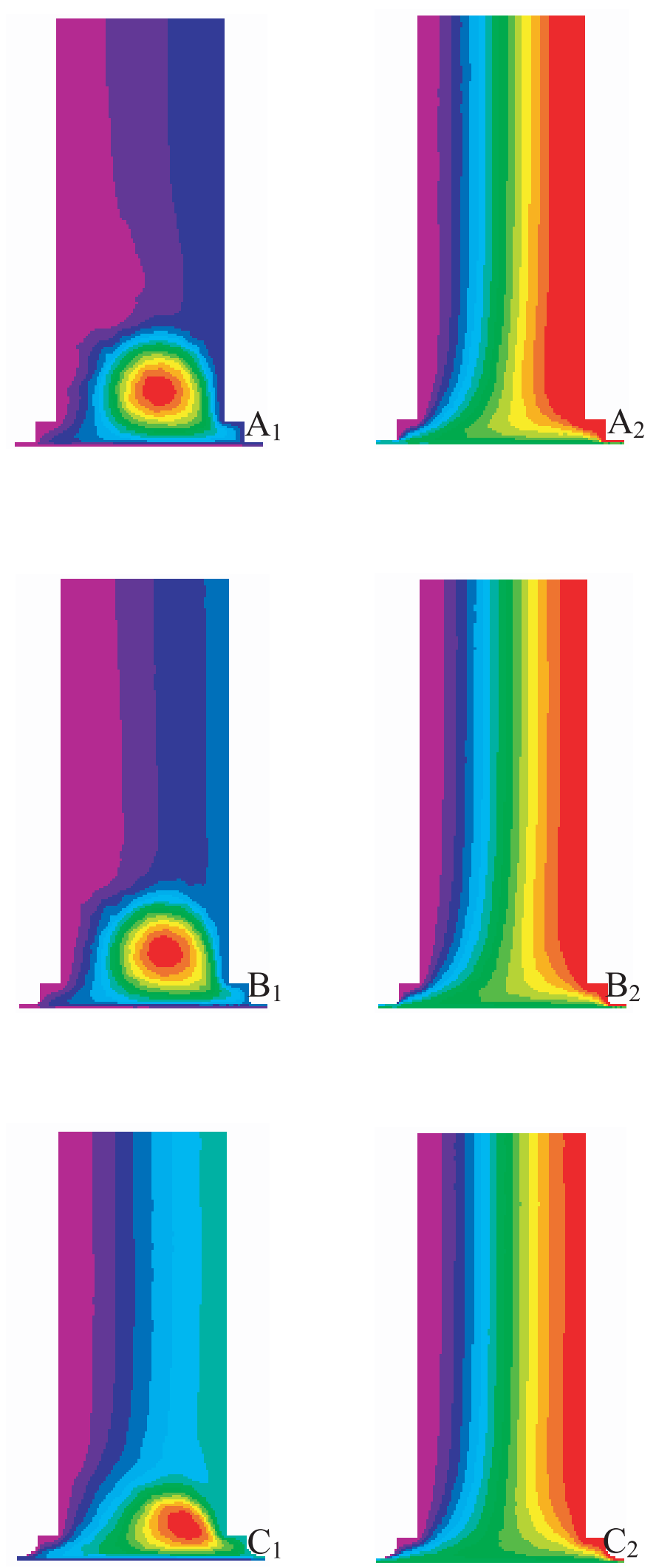

Fig. 3. Lignes de courant dans la partie centrale de la rainure et dans le trou d'alimentation pour différentes formes du raccordement rainure/coussinet $\left(\mathrm{A}_{1}\right.$ et $\mathrm{A}_{2}: \mathrm{RRC}$ carré, $\mathrm{B}_{1}$ et $\mathrm{B}_{2}$ : $R=0,8 \mathrm{~mm}$ et $\mathrm{C}_{1}$ et $\left.\mathrm{C}_{2}: R=1,6 \mathrm{~mm}\right) . U_{\text {arb }}=\left(3,4 \mathrm{~m} \cdot \mathrm{s}^{-1}\right.$ et $\left.1 \mathrm{~m} \cdot \mathrm{s}^{-1}\right) ; P_{\text {alim }}=(0,04 \mathrm{MPa}$ et $0,2 \mathrm{MPa})$. 


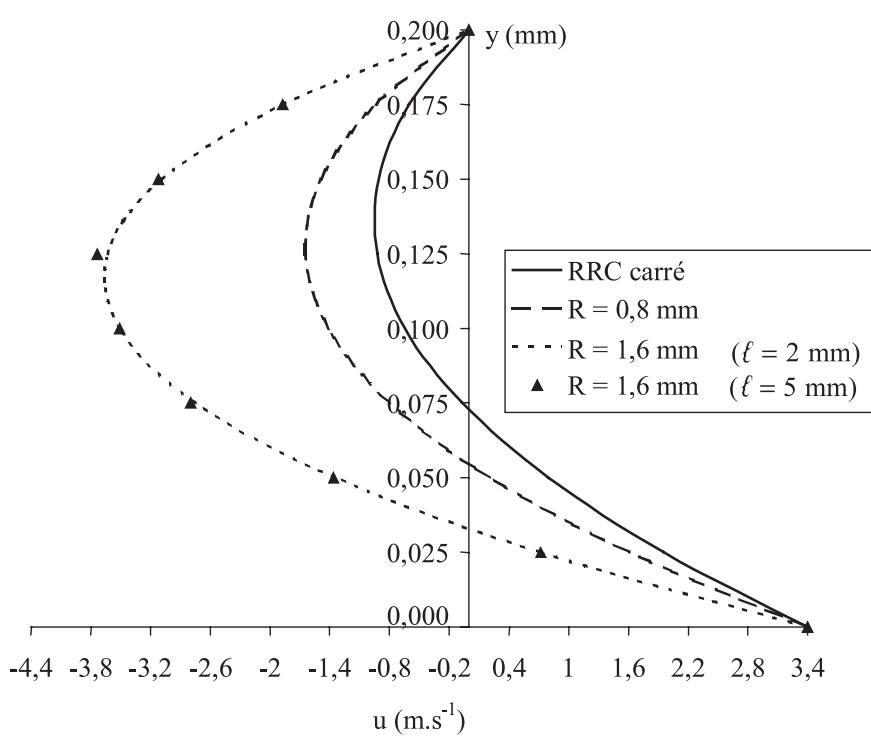

Fig. 4. Profil de vitesse dans la section amont du lubrifiant refoulé à contre-courant pour différentes formes du raccordement rainure/coussinet $\left(U_{\text {arb }}=3,4 \mathrm{~m} \cdot \mathrm{s}^{-1}\right.$ et $\left.P_{\text {alim }}=0,04 \mathrm{MPa}\right)$.

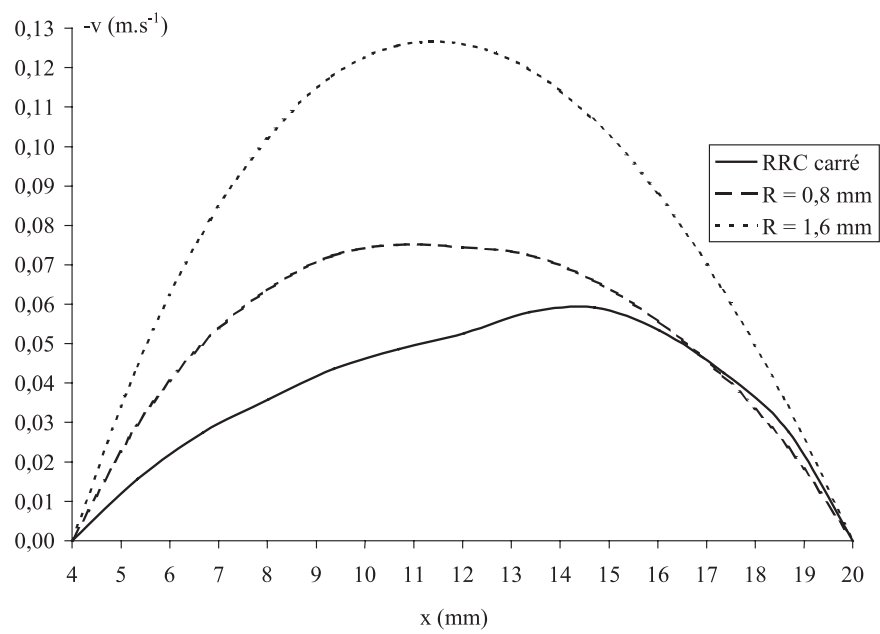

Fig. 5. Profil de vitesse dans la section $y=h_{\mathrm{t}} / 2$ du trou d'alimentation pour différentes formes du raccordement rainure/coussinet $\left(U_{\text {arb }}=3,4 \mathrm{~m} \cdot \mathrm{s}^{-1}\right.$ et $\left.P_{\text {alim }}=0,04 \mathrm{MPa}\right)$.

domaine de sortie $(\ell=2 \mathrm{~mm})$ est suffisante pour que l'écoulement soit établi quel que soit RRC. La figure 4 montre un exemple de calcul pour le cas $R=1,6 \mathrm{~mm}$ et $\ell=5 \mathrm{~mm}$.

En ce qui concerne le conduit d'alimentation, le profil de vitesse dans la section $y=h_{\mathrm{t}} / 2$ est très proche d'un profil parabolique pour $R=1,6 \mathrm{~mm}$ (Fig. 5). La perturbation de l'écoulement dans le conduit d'alimentation due à l'effet de la vitesse de l'arbre est donc moins importante dans le cas d'une rainure suffisamment profilée.

L'effet de Couette dominant $\left(U_{\text {arb }}=3,4 \mathrm{~m} \cdot \mathrm{s}^{-1}\right.$ et $\left.P_{\text {alim }}=0,04 \mathrm{MPa}\right)$ cause la diminution de la taille du tourbillon dans le cas où le rayon de courbure de RRC est le plus grand (Fig. $3 \mathrm{C}_{1}$ ). L'augmentation de $R$ contribue également à une baisse de la résistance à l'écoulement dans ces régions et donc à l'augmentation

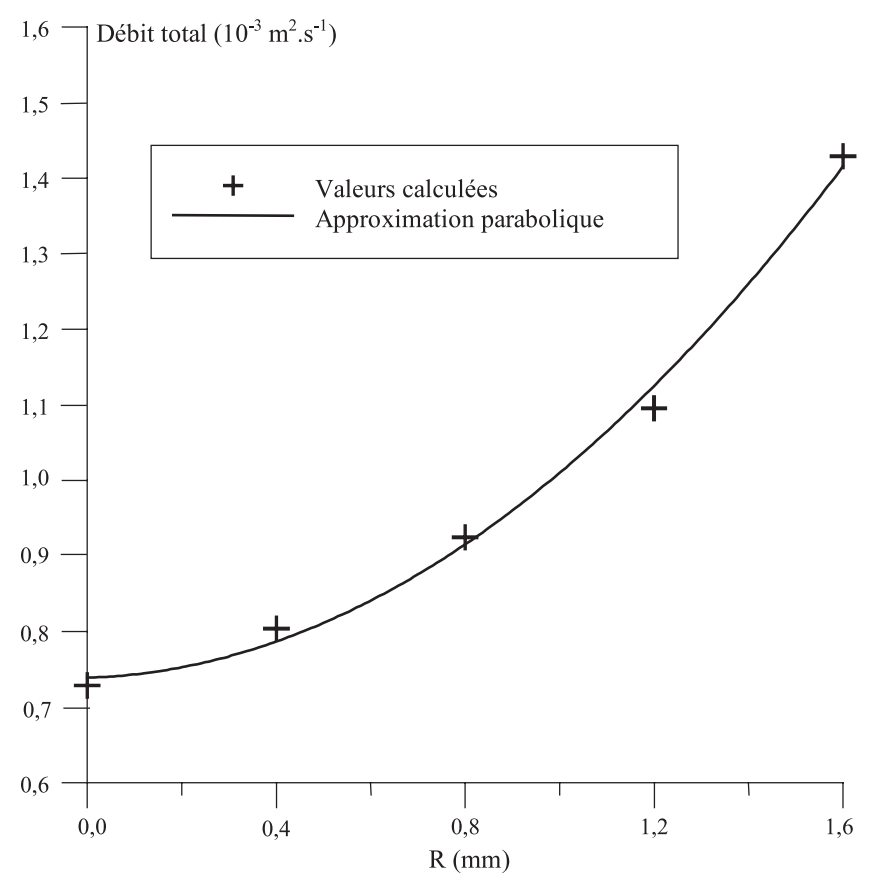

Fig. 6. Influence du rayon de courbure $R$ sur le débit total $\left(U_{\text {arb }}=3,4 \mathrm{~m} \cdot \mathrm{s}^{-1}\right.$ et $\left.P_{\text {alim }}=0,04 \mathrm{MPa}\right)$.

Tableau 3. Débit unitaire $\left(10^{-3} \mathrm{~m}^{2} \cdot \mathrm{s}^{-1}\right)$, cas d'un effet de Couette dominant.

\begin{tabular}{lccl}
\hline & $\begin{array}{c}\text { Débit sortant } \\
\text { de la rainure } \\
\text { vers l'amont }\end{array}$ & $\begin{array}{c}\text { Débit sortant } \\
\text { de la rainure } \\
\text { vers l'aval }\end{array}$ & Total \\
\hline RRC carré & 0,0725 & 0,6561 & 0,7286 \\
$R=0,4 \mathrm{~mm}$ & 0,1044 & 0,6992 & 0,8036 \\
$R=0,8 \mathrm{~mm}$ & 0,1593 & 0,7654 & 0,9247 \\
$R=1,2 \mathrm{~mm}$ & 0,2437 & 0,8516 & 1,0953 \\
$R=1,6 \mathrm{~mm}$ & 0,3963 & 1,0326 & $1,4289(+96 \%)$ \\
\hline
\end{tabular}

du débit unitaire $q$ sortant sur les deux faces, gauche et droite, de la rainure (Tab. 3). Le débit total varie paraboliquement avec le rayon de courbure $R$ (Fig. 6).

\section{3 Écoulements dans la rainure avec effet de la pression d'alimentation dominant $\left(U_{\text {arb }}=1 \mathrm{~m} . \mathrm{s}^{-1}\right.$ et $\left.P_{\text {alim }}=0,2 \mathrm{MPa}\right)$}

La figure 3 représente une comparaison des lignes de courant dans la région centrale de la rainure dans le cas d'un effet de Couette dominant (Figs. $3 \mathrm{~A}_{1}-3 \mathrm{C}_{1}$, avec $U_{\text {arb }}=3,4 \mathrm{~m} \cdot \mathrm{s}^{-1}$ et $\left.P_{\text {alim }}=0,04 \mathrm{MPa}\right)$ et dans le cas d'une pression d'alimentation dominante (Figs. $3 \mathrm{~A}_{2}-3 \mathrm{C}_{2}$, avec $U_{\text {arb }}=1 \mathrm{~m} . \mathrm{s}^{-1}$ et $\left.P_{\text {alim }}=0,2 \mathrm{MPa}\right)$. RRC varie d'une forme carrée à une forme arrondie avec $R=0,8 \mathrm{~mm}$ et $R=1,6 \mathrm{~mm}$, respectivement. La différence qualitative majeure entre les deux cas est la suppression totale du tourbillon dans le cas d'une pression d'alimentation dominante, et une disparition de la zone de recirculation dans les deux parties, gauche et droite, de la rainure relative aux trois formes de RRC comme le montre les figures $7 \mathrm{~A}_{2}-7 \mathrm{C}_{2}$. 

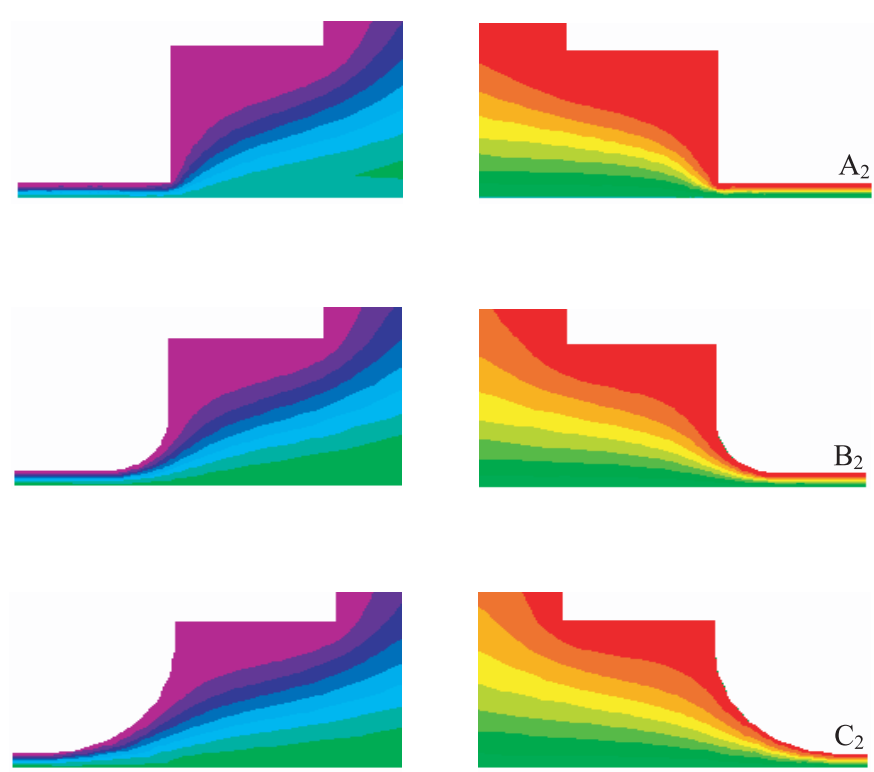

Fig. 7. Lignes de courant dans la rainure pour différentes formes du raccordement rainure/coussinet $\left(U_{\mathrm{arb}}=1 \mathrm{~m} . \mathrm{s}^{-1}\right.$ et $\left.P_{\text {alim }}=0,2 \mathrm{MPa}\right) . \mathrm{A}_{2}: \mathrm{RRC}$ carré, $\mathrm{B}_{2}: R=0,8 \mathrm{~mm}$ et $\mathrm{C}_{2}$ : $R=1,6 \mathrm{~mm}$.

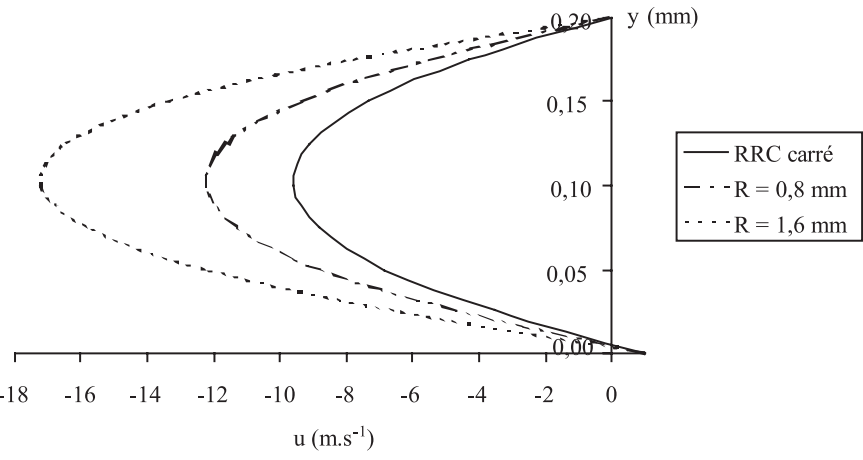

Fig. 8. Profil de vitesse dans la section amont du lubrifiant refoulé à contre-courant pour différentes formes du raccordement rainure/coussinet $\left(U_{\text {arb }}=1 \mathrm{~m} \cdot \mathrm{s}^{-1}\right.$ et $\left.P_{\text {alim }}=0,2 \mathrm{MPa}\right)$.

La figure 8 représente l'effet de l'augmentation du rayon de courbure de RRC sur le profil de vitesse dans la section d'entrée du lubrifiant refoulé à contre-courant. L'effet de la pression d'alimentation est très dominant par rapport à celui du mouvement de l'arbre. La quantité de fluide sortant devient de plus en plus grande avec l'augmentation du rayon de courbure de RRC.

La variation de RRC d'une forme carrée à une forme arrondie avec $R=0,8 \mathrm{~mm}$ et $R=1,6 \mathrm{~mm}$ cause l'augmentation du débit unitaire sortant sur les deux faces, gauche et droite, de la rainure (Tab. 4).

\subsection{Profil de pression pour différentes formes de RRC}

La figure 9 représente le profil de pression sur l'arbre, pour différentes formes de RRC, dans le cas d'un écoulement de Couette dominant lorsque $U_{\text {arb }}=$ $3,4 \mathrm{~m} . \mathrm{s}^{-1}$ et $P_{\text {alim }}=0,04 \mathrm{MPa}$. Le changement brutal de
Tableau 4. Débit unitaire $\left(10^{-3} \mathrm{~m}^{2} \cdot \mathrm{s}^{-1}\right)$, cas d'une pression d'alimentation dominante.

\begin{tabular}{lccl}
\hline & $\begin{array}{c}\text { Débit sortant } \\
\text { de la rainure } \\
\text { vers l'amont }\end{array}$ & $\begin{array}{c}\text { Débit sortant } \\
\text { de la rainure } \\
\text { vers l'aval }\end{array}$ & Total \\
\hline RRC carré & 1,2451 & 1,4093 & 2,6544 \\
$R=0,4 \mathrm{~mm}$ & 1,3975 & 1,5575 & 2,9550 \\
$R=0,8 \mathrm{~mm}$ & 1,5971 & 1,7495 & 3,3466 \\
$R=1,2 \mathrm{~mm}$ & 1,8710 & 2,0106 & 3,8816 \\
$R=1,6 \mathrm{~mm}$ & 2,2564 & 2,4264 & $4,6828(+76 \%)$ \\
\hline
\end{tabular}

la direction de l'écoulement au niveau de la région de sortie de la rainure est responsable de l'augmentation locale de la pression en amont de ce point suivi par une chute rapide de celle-ci en raison des pertes de charge par frottement visqueux dans la configuration RRC carrée. En présence d'un grand tourbillon, le développement d'une pression élevée entre l'arbre et le tourbillon est nécessaire pour pouvoir entraîner le lubrifiant à l'extérieur de la rainure.

Sur la figure 10 et pour les mêmes formes de RRC mais dans le cas d'une pression d'alimentation dominante $\left(U_{\text {arb }}=1 \mathrm{~m} . \mathrm{s}^{-1}\right.$ et $\left.P_{\text {alim }}=0,2 \mathrm{MPa}\right)$, on observe une distribution presque symétrique de la pression au niveau de l'arbre. Ce profil de pression est justifié si l'on observe les lignes de courant illustrées sur la figure 7 , où l'écoulement est sortant sur les deux faces, gauche et droite, de la rainure.

\section{Conclusion}

Une simulation numérique a été développée pour analyser les effets de la forme du raccordement rainure/coussinet d'un palier hydrodynamique sur la physique de l'écoulement du lubrifiant aussi bien dans le cas d'un effet de Couette dominant que dans le cas d'une pression d'alimentation dominante. Les équations bidimensionnelles de Navier-Stokes sont résolues numériquement par la méthode des éléments finis en utilisant la méthode de chargement par incrémentation.

Les résultats montrent que l'effet de Couette dominant génère une augmentation de la pression dans la région droite de la rainure. Cette pression, nécessaire pour entraîner le lubrifiant à l'extérieur de la rainure, diminue avec l'augmentation du rayon de courbure de RRC. Par contre, la pression est relativement uniforme dans la rainure dans le cas d'une pression d'alimentation dominante.

En particulier, il est établi par cette simulation que le débit d'alimentation en lubrifiant peut être augmenté de $96 \%$ (cas d'un effet de Couette dominant) et de $76 \%$ (cas d'une pression d'alimentation dominante) sans changer la pression d'alimentation lorsque la rainure est suffisamment profilée.

Remerciements. Nous remercions le Comité Mixte Interuniversitaire Franco-Marocain pour son soutien de ce travail, effectué dans le cadre du programme de l'Action Intégrée $\mathrm{N}^{\circ}: \mathrm{MA} / 03 / 80$. 


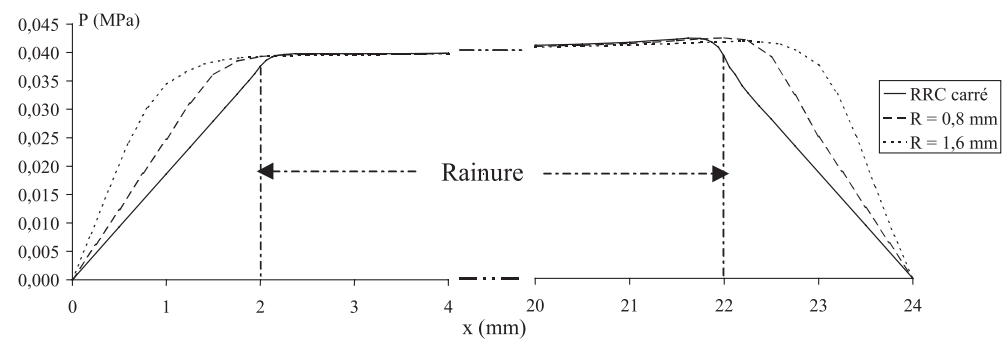

Fig. 9. Distribution de la pression sur l'arbre pour différentes formes du raccordement rainure $/{\text { coussinet }\left(U_{\text {arb }}=3,4 \text { m.s }\right.}^{-1}$ et $\left.P_{\text {alim }}=0,04 \mathrm{MPa}\right)$.

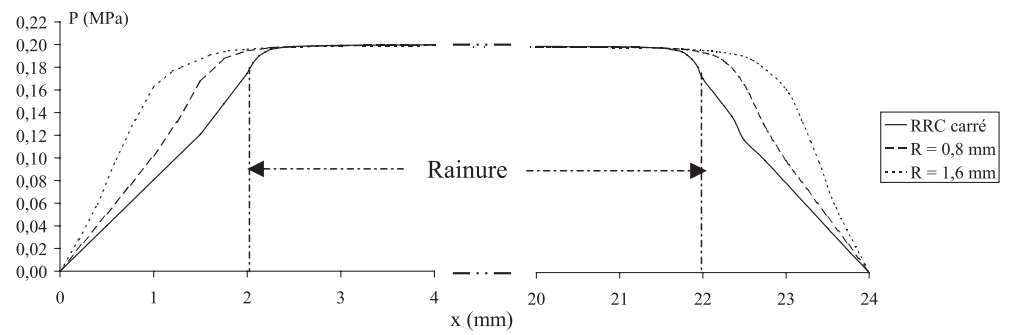

Fig. 10. Distribution de la pression sur l'arbre pour différentes formes du raccordement rainure $/$ coussinet $\left(U_{\text {arb }}=1 \mathrm{~m} . \mathrm{s}^{-1}\right.$ et $\left.P_{\text {alim }}=0,2 \mathrm{MPa}\right)$.

\section{Références}

[1] D.M. Pirro, A.A. Wessol, Lubrication fundamentals, Second edition, Mechanical engineering series 137, 2001

[2] J. Frêne, D. Nicolas, B. Degueurce, D. Berthe, M. Godet, Lubrification hydrodynamique - Paliers et Butées, Collection de la DER EDF, Eyrolles, 1990

[3] C. Ettles, Solutions of flow in a bearing groove, Proc. Inst. Mech. Eng. 182, 1968, 122-133

[4] D.D. Heckelman, C. Ettles, Viscous and inertial pressure effects at the inlet of a bearing film, ASLE Preprint 87AM-2A-1, 1987

[5] J.A. Tichy, P. Bourgin, The effect of inertia in lubrication flow including entrance and initial conditions, J. Appl. Mech. 52 (1985) 759-765

[6] M.J. Braun, F.K. Choy, Y.M. Zhou, The effects of a hydrostatic pocket aspect ratio and its supply orifice position and attack angle on steady state flow patterns, pressure and shear characteristics, ASME J. Tribology 115 (1993) 678-685

[7] M.J. Braun, Y.M. Zhou, F.K. Choy, Transient flow patterns and pressures characteristics in a hydrostatic pocket, ASME J. Tribology 116 (1994) 139-146

[8] M. Dzodzo, M.J. Braun, R.C. Hendricks, Pressure and flow characteristics in a shallow hydrostatic pocket with rounded pocket/land joints, in : International Tribology Conference-AUSTRIB, 1994, pp. 587-592

[9] O.C. Zienkiewicz, R.L. Taylor, The finite element method, Fourth edition, 2, Chap. 3, McGraw-Hill, London, 1989

[10] O.C. Zienkiewicz, R.L. Taylor, The finite element method, Fourth edition, 1, Chap. 13, McGraw-Hill, London, 1989 